Revue internationale P.M.E.

Économie et gestion de la petite et moyenne entreprise

\title{
Les politiques de formation dans les PME-PMI françaises : l'émergence de pratiques novatrices
}

\section{André Rosanvallon}

Volume 3, numéro 1, 1990

URI : https://id.erudit.org/iderudit/1007947ar

DOI : https://doi.org/10.7202/1007947ar

Aller au sommaire du numéro

Éditeur(s)

Presses de l’Université du Québec

ISSN

0776-5436 (imprimé)

1918-9699 (numérique)

Découvrir la revue

Citer cet article

Rosanvallon, A. (1990). Les politiques de formation dans les PME-PMI

françaises : l'émergence de pratiques novatrices. Revue internationale P.M.E.,

3(1), 75-87. https://doi.org/10.7202/1007947ar
Résumé de l'article

L'article rend compte des premiers résultats d'une recherche ayant pour thème les politiques de formation continue dans les PME-PMI de la région Rhône-Alpes. L'accent est mis sur la description et l'analyse de pratiques de formation innovantes qui témoignent de l'émergence de nouvelles modalités d'apprentissage au sein des PME-PMI et de nouveaux modes d'insertion de celles-ci dans les réseaux externes de compétence en matière de formation. Par delà la portée et la pertinence des innovations, l'article rappelle différentes limites qui provoquent des interrogations sur le caractère significatif des transformations en cours. 


\title{
Les politiques de formation dans les PME-PMI françaises: l'émergence de pratiques novatrices
}

\author{
André ROSANVALLON \\ Université des Sciences Sociales de Grenoble
}

\begin{abstract}
RÉSUMÉ
L'article rend compte des premiers résultats d'une recherche ayant pour thème les politiques de formation continue dans les PME-PMI de la région Rhône-Alpes. L'accent est mis sur la description et l'analyse de pratiques de formation innovantes qui témoignent de l'émergence de nouvelles modalités d'apprentissage au sein des PME-PMI et de nouveaux modes d'insertion de celles-ci dans les réseaux externes de compétence en matière de formation. Par delà la portée et la pertinence des innovations, l'article rappelle différentes limites qui provoquent des interrogations sur le caractère significatif des transformations en cours.
\end{abstract}

\begin{abstract}
The author provides an account of the initial results of a study of further education policies among small to medium-sized firms in the Rhône-Alpes region. Emphasis is placed on the description and analysis of innovatory training practices (signs of the emergence of new learning methods in such firms), and of new ways of integrating these with other external networks of training skills. After dealing with the extent and relevance of the innovations, the author points out various limits to the significance of the changes currently taking place.
\end{abstract}

\section{RESUMEN}

El articulo de los primeros resultados de una investigacion cuyos temas son las politicas de formacion continua en las PyME de la region Rhône-Alpes. Se insiste en la descripcion y en el analisis de las practicas de formacion innovadoras que son el testigo de la emergencia de nuevas modalidades de aprendizaje en las PyME y de nuevos modos de insercion de estas formaciones en las redes externas de formacion. Mas alla del alcance y de la pertinencia de las innovaciones el articulo recuerda distintos limites que interrogan sobre el caracter significativo de las transformaciones existentes.

- Docteur d'État en économie et ingénieur de Recherche à l'Institut de recherche économique production développement, Université des Sciences Sociales de Grenoble. Ses champs des recherches sont l'analyse des politiques de formation dans les entreprises en relation avec les mutations en cours dans le système productif. Il est l'auteur de plusieurs publications en la matière. Adresse: 3, Allée des Vosges, 38130 ECHIROLLES, France. 


\section{Introduction}

On assiste actuellement à de profondes mutations dans la manière dont les entreprises en France gèrent et développent la formation de leur main-d'oeuvre. Cela se vérifie particulièrement dans le cas des grands groupes industriels, comme en témoignent un certain nombre de recherches récentes(Mehaut, 1989). Dans le cas des PME-PMI, les informations disponibles sont beaucoup plus fragmentaires et parcellaires. Au sein de l'importante littérature consacrée à l'analyse des dynamismes qui traversent ce secteur, la composante formation-emploi est rarement prise en compte. Quand elle l'est, on se réfere le plus souvent à un système de formation dominant au sein des PME-PMI, auteur de modalités plus ou moins progressives d'apprentissage sur le tas (Gehin, 1986).

Depuis quelque temps cependant, plusieurs familles d'informations (Lutz, 1983: Fougère, 1986) suggèrent l'émergence de nouvelles approches de formation au sein des PME-PMI, conformes aux spécificités de ces entreprises (en terme d'atouts et d'handicaps) et plus proches des «modèles de formation» des grandes entreprises, par un recours croissant à des procédures plus formalisées d'extériorisation de la formation.

Pour vérifier la pertinence de ces propos et pour les affiner, une recherche est en cours, financée par les autorités de la région Rhône-Alpes en France. Son objet vise à identifier, décrire et analyser ces pratiques innovantes en matière de formation pour mettre en évidence en quoi elles sont significatives de mutations dans les processus d'apprentissage au sein des PME-PMI, et en quoi elles sont ou non reproductibles.

\section{$2 \quad$ La méthode retenue}

Dans la délimitation du champ d'observation et d'analyse, nous avons volontairement privilégié les petites entreprises occupant plus d'une vingtaine et moins d'une centaine de salariés et ayant une gestion autonome, non dépendante d'une société mère. Par ailleurs, nous nous sommes écartés d'une vision trop restrictive des innovations. Sachant que celles-ci ont un caractère progressif, le plus souvent partiel et transitoire, nous avons retenu comme pratiques de formation innovantes, celles qui ne sont pas conformes aux pratiques ordinaires (quelle que soit l'ampleur de l'écart ou de la déviation) de l'avis de ceux qui ont à connaître de la diversité des situations de formation au sein des PME-PMI.

Notre méthode repose sur des enquêtes directes auprès de PME-PMI implantées dans la région Rhône-Alpes et ayant développé des pratiques de formation originales par rapport aux pratiques standards, en collaboration avec des organismes de formation qui interviennent dans l'environnement de ces entreprises (ASFO,FAF, Centres de formation...). Dans cette perspective, un comité de pilotage 
a été constitué dès le départ de la recherche, associant des organismes de formation (privés ou publics) et des organismes de gestion et d'administration de la formation (publics ou privés). Ce comité de pilotage s'est réuni une première fois pour la mise en place du dispositif de la recherche; une seconde fois, pour sélectionner les exemples qui apparaissaient significatifs à l'ensemble des membres du collectif. Depuis, le comité assure un suivi régulier de la recherche.

La recherche s'appuie sur la réalisation de monographies autour des PMEPMI préalablement inventoriées et sélectionnées. Mais comment identifier des PMEPMI qui innovent sachant que, par définition, cette notion recouvre une réalité floue, difficile à caractériser par des indicateurs pertinents ? Pour parvenir à cet inventaire nous avons eu recours à plusieurs modalités d'enquête, sachant à priori que nous ne pourrions pas parvenir à l'exhaustivité. Premièrement, nous avons enquêté de manière assez systématique auprès de différents interlocuteurs qui, de par leur domaine de compétences, étaient susceptibles de nous indiquer le nom d'entreprises innovantes en matière de formation. L'enquête a concerné en particulier des organismes de formation privés et publics ainsi que des organismes de gestion et de conseil implantés dans la région. Ensuite, cette enquête a été complétée par une interrogation plus large, sur les entreprises innovantes régionales dans le domaine technologique ou organisationnel (en faisant l'hypothèse que ces entreprises étaient plus à même d'innover en formation que les autres) à partir du traitement de fichiers d'établissements existants, et d'un dépouillement de la presse spécialisée sur la vie des entreprises. Sur la base de ces différents questionnements, nous avons recensé une première liste d'une cinquantaine d'entreprises susceptibles d'être intégrées dans un échantillon de PME innovantes en matière de formation.

La sélection des expériences les plus intéressantes du point de vue de la problématique de la recherche a été assurée au sein du comité de pilotage précédemment signalé. Dans le cadre de la première phase de la recherche, sept d'entre elles, significatives des innovations en cours dans le domaine de la formation dans les PME-PMI ont été retenues dont cinq cas qui concernent des entreprises, les deux autres cas étant relatifs à l'offre de formation.

\subsection{Les entreprises innovantes enquêtées}

L'entreprise A, du secteur de la construction mécanique, occupe 35 personnes. L'introduction de nouvelles commandes numériques s'accompagne d'un plan de formation des opérateurs, qui innove sur plusieurs plans. L'apprentissage à la maîtrise des nouveaux équipements ne se limite pas à un simple transfert de savoirfaire de manipulation assuré par les instructeurs envoyés par le constructeur au moment de la phase d'installation. L'entreprise alterne formation pratique-formation théorique, en combinant l'offre publique de formation à la mobilisation des compétences du fabricant. Dans un premier temps, le lycée technique implanté dans le voisinage de l'usine assure la formation des futurs opérateurs en la centrant sur le fonctionnement théorique des nouvelles commandes numériques. Dans un second 
temps, les mêmes enseignants sont associés à la formation pratique animée par les instructeurs du constructeur. Partant des connaissances théoriques initialement acquises par les opérateurs, les enseignants du lycée assurent l'interface avec les instructeurs pour permettre un transfert effectif des qualifications auprès des futurs opérateurs.

L'entreprise B a des activités «traditionnelles» de fabrication de classeurs et de dossiers en carton. Elle emploie 65 personnes. Le contexte dans lequel s'inscrit les innovations de formation est assez semblable au cas précédent quant à l'introduction d'un processus d'automatisation. Elles se manifestent sous la forme d'une construction plus formalisée des actions de formation, sans que celles-ci soient déconnectées de la réalité des modes opératoires effectifs. Bien au contraire, la formation est un instrument privilégié pour «théoriser» les dysfonctionnements particulièrement nombreux au moment de la phase d'implantation des équipements et de montée en production. Elle vise en même temps à mémoriser l'expérience progressivement acquise, sous la forme de manuels qui serviront ensuite de supports pédagogiques pour améliorer les processus d'apprentissage sur le tas. Dans le cas des entreprises $\mathrm{A}$ et $\mathrm{B}$ il y a un mouvement cumulatif d'innovations qui concernent à la fois les stratégies de formation et les «outils et supports pédagogiques» en tenant compte des spécificités des PME-PMI.

Dans le cas des entreprises $\mathrm{C}$ et $\mathrm{D}$, on a plutôt affaire à des pratiques de formation articulées à des innovations organisationnelles. L'entreprise $C$ (98 personnes) est spécialisée dans des activités voisines de celles de l'entreprise B, pour la production d'articles de bureaux et de fournitures scolaires. La formation intervient comme outil stratégique d'un management participatif orienté vers la définition d'un projet d'entreprise. Le plan de formation pluri-annuel est organisé autour de la mise en oeuvre de nouveaux rapports de partenariat entre l'entreprise et l'offre de formation à travers l'établissement de relations croisées et complémentaires, visant à combiner en permanence l'acquisition de savoirs empiriques et de savoirs plus formalisés. Cet exemple est significatif de l'émergence de nouvelles capacités des PME-PMI à s'insérer dans des réseaux externes de compétence en matière de formation.

L'entreprise $D$ est une PME de moins de 20 salariés, mais particulièrement performante dans la fabrication de moules plastiques. La formation est construite en cohérence avec l'organisation du travail. Ainsi la formation sous la forme d'actions explicites, s'accompagne de mesures visant à rendre l'organisation du travail plus pédagogique et plus formatrice (à travers la recomposition des collectifs de travail associant les opérateurs et les programmeurs et l'organisation quasi systématique de phases de chevauchement entre les équipes). Le cas de cette entreprise nous a permis par ailleurs d'observer un cas de partenariat en terme de formation avec une partie des principaux donneurs d'ordre.

La cinquième expérience (cas $\mathrm{E}$ ) qui a fait l'objet d'observations minutieuses au cours de notre enquête est une opération inter-entreprises entre sept PMI 
implantées sur un même technopole et ayant toutes des activités dans des domaines de technologie de pointe (informatique, micro-électronique, automatisme...) et étant toutes concernées par des problèmes d'industrialisation et de gestion de la production. Cette expérience qui repose sur des principes de regroupement et de coordination, est articulée autour de deux sous-ensembles d'actions de formation: un tronc commun constitué d'actions inter-entreprises d'une part, des actions spécifiques aux besoins propres de chaque entreprise d'autre part. Comme les précédentes - mais selon des modalités particulières - cette expérience témoigne d'innovations dans les pratiques de formation continue bien adaptées et conformes aux spécificités des PME-PMI, eu égard à leur taille.

\subsection{Deux cas de pratiques innovantes dans l'environnement des PME-PMI}

Le premier cas étudié (cas F) concerne le lancement et la mise en oeuvre d'un service commun à l'initiative conjointe du service public de l'emploi (par l'intermédiaire de la Direction départementale du travail et de l'emploi) et d'une organisation professionnelle décentralisée, et qui a pour vocation d'apporter conseil et assistance aux PME-PMI régionales en matière de formation. La création de ce service témoigne de l'existence de nouvelles combinaisons et de nouvelles complémentarités entre le service privé et le service public en matière d'aide, d'assistance et de conseil pour améliorer l'efficacité de l'offre de formation en direction des PME-PMI. Elle est par ailleurs significative d'un élargissement des domaines d'intervention de ces organismes par une intégration croissante des activités d'amont dans la phase d'analyse des besoins et de l'ingénierie de formation.

Le second cas (cas $\mathrm{G}$ ) est relatif aux modalités de fonctionnement d'un organisme original, à l'interface entre l'offre et la demande de formation, et qui associe différents partenaires dont les collectivités locales. Par là même, cet organisme est l'expression d'une volonté de renforcer l'outil de formation comme instrument de structuration du tissu industriel autour des PME-PMI et comme lieu du développement local, à l'initiative des collectivités décentralisées.

\section{L'ampleur des dépenses de formation déclarées par les PME-PMI dans la région Rhône-Alpes}

Les déclarations annuelles des entreprises au Trésor font l'objet d'un traitement statistique au niveau régional qui nous renseigne sur le montant et la structure des dépenses dans le cadre de l'obligation légale de financement de la formation. L'analyse des résultats de ce traitement suggère les commentaires suivants.

Tout d'abord, ils mesurent la faiblesse des dépenses de formation parmi les PME-PMI de la région. Dans les entreprises occupant de 10 à 19 salariés, la formation 
représente $1,2 \%$ du montant de la masse salariale. La proportion est de $1,23 \%$ pour celles dont la taille est comprise entre 20 et 49 salariés. Près de deux PME-PMI sur trois occupant moins de 50 salariés s'en tiennent à un budget de formation correspondant juste à l'obligation légale. L'effort est près de 2,5 fois moins important, en valeur relative, que dans les grandes entreprises régionales de plus de 2000 salariés.

Dans le temps, on observe une relative stabilité de l'effort de formation au sein des PME-PMI régionales, autour de phases contrastées: une réduction sensible de cet effort de 1983 à 1985, puis une reprise après cette date qui semble s'amplifier depuis. Dans la même période, le montant des versements au Trésor (correspondants à la part de l'obligation légale non dépensée en formation) n'a cessé de décroître. En 1986, les reversements au Trésor ne représentent plus que $2 \%$ des dépenses effectives de formation parmi les entreprises de 20 à 49 salariés. En ce qui concerne les bénéficiaires de la formation, il apparaît que dans les PME-PMI de la région environ 1 salarié sur 10 dans les entreprises de moins de 50 salariés bénéficie d'un stage de formation au cours de l'année. Cette situation moyenne recouvre des probabilités d'accès à la formation différentes selon les catégories socio-professionnelles qui tendent à accentuer les disparités entre les niveaux de qualifications de la maind'oeuvre, plutôt qu'à les réduire.

De toute évidence, ces données sont à utiliser avec une très grande prudence. En effet, on connaît la nécessité de distinguer plusieurs types de comptabilisation des efforts de formation entre ce qui est déclaré, négocié, formalisé par rapport aux dépenses effectives. On doit rappeller aussi que ce traitement statistique de l'effort de formation des entreprises rend très mal compte de la réalité des formations au sein des PME-PMI, en ne comptabilisant que les actions sous la forme de stages, au détriment des formations sur le tas qui constituent souvent l'essentiel de la formation. Enfin, ces données sont incapables, par définition, de saisir les dynamiques et les évolutions en cours dans les pratiques de formation des entreprises.

Nous aurons cependant à nous rappeler ultérieurement que s'il existe des expériences de formation innovantes au sein des PME-PMI, celles-ci se développent dans un contexte où l'ampleur de l'effort de formation correspond mal aux enjeux que recouvre la production de nouvelles qualifications pour faire face aux mutations actuelles, qu'elles soient technologiques ou organisationnelles.

\section{$4 \quad$ Le constat de pratiques novatrices en matière de formation pour et par les PME-PMI}

Bien qu'inachevée, la recherche en cours a déjà permis d'identifier et d'analyser un premier ensemble de pratiques de formation innovantes. Celles-ci s'observent à plusieurs niveaux. Certaines apparaissent au plan interne des entreprises, dans leur manière d'appréhender leurs besoins de formation et d'y répondre. D'autres se manifestent davantage dans l'environnement des PME-PMI, en terme de 
gestion externe de la formation en direction de ces entreprises. Dans la majorité des cas que nous avons étudiés, ce qui est frappant, c'est la manière dont se combinent et se complètent ces différentes innovations pour donner une efficience plus grande aux stratégies de formation pour et par les PME-PMI.

\subsection{Les innovations dans les modes de gestion de la formation, internes aux PME-PMI}

Faute d'un recensement suffisamment exhaustif, il est difficile de parvenir à une typologie pertinente de ces innovations. Nos premiers constats témoignent cependant de l'existence d'innovations multiformes et originales qui s'inscrivent dans un processus plus large de transformation des modes de gestion des ressources humaines au sein des PME-PMI.

Il ressort de notre enquête une remise en cause d'un modèle traditionnel d'apprentissage dans les PME-PMI centré de manière quasi exclusive sur l'expérience acquise sur le tas. Actuellement on assiste à des modifications sensibles dans les processus généraux d'apprentissage au sein des PME-PMI tant au niveau du contenu des sous-ensembles qui les constituent qu'au niveau de leurs modes d'articulation. En particulier, on constate une amplification sensible des modalités de transmission et d'acquisition des connaissances sous la forme de stages qui accompagnent une modernisation des processus d'apprentissage plus traditionnels. Cette tendance à une intensification des formations sous la forme de stages - au sens où ils sont définis dans le dispositif juridique de la formation professionnelle - est la plus manifeste. L'image de la PME-PMI qui, en matière de formation, se limiterait au minimum légal obligatoire ne peut être généralisée de manière aussi systématique qu'on a pu le faire auparavant. Même si en moyenne les dépenses déclarées sont proches de l'obligation légale, dans un certain nombre de cas qui n'apparaissent pas tout à fait exceptionnels, l'effort de formation des PME-PMI se rapproche ou dépasse ce qui se fait dans les grandes entreprises. Dans les PME-PMI de l'opération interentreprises (cas E), la formation est comprise dans une fourchette de 6 à $10 \%$ de la masse salariale. Cette situation n'est pas propre aux PME-PMI qui sont placées sur le créneau des technologies très avancées. Dans le cas d'entreprises spécialisées dans des activités plus traditionnelles, l'effort peut être tout aussi remarquable. Ainsi dans l'entreprise D (moules plastiques) plus de $10 \%$ de la masse salariale est consacré à la formation.

L'envoi de salariés de PME-PMI en stage dans des organismes de formation externes n'est pas en soi une nouveauté. Ce qui apparaît caractéristique des innovations en cours, c'est le développement de stages en interne, conçus sur mesure et qui intègrent les spécificités de l'entreprise tant dans la définition du contenu des formations que dans le choix des modalités de leur mise en oeuvre. Ainsi, ce qui est en jeu en terme d'innovation, ce n'est pas seulement la multiplication et la diversification des formations sous la forme de stage, c'est surtout la manière dont ceux-ci recomposent les processus plus conventionnels d'apprentissage au sein des PME- 
PMI. En ce sens, plusieurs exemples témoignent de modifications en cours des procédures informelles d'apprentissage sur le tas en liaison avec le développement de stages «formalisés»: stages prenant pour support pédagogique les modalités concrètes de fonctionnement du procès de travail (entreprise B), stages ayant pour objectif l'acquisition d'une nouvelle culture d'entreprise (entreprise C) ou de nouvelles méthodes de résolution des problèmes dans la formation de chefs de projets (cas C).

D'autres cas sont encore plus significatifs d'innovations en cours dans les modalités d'apprentissage à la maîtrise des innovations techniques, par rapport aux formations «traditionnelles» des constructeurs (entreprises A, B, D...). Elles se manifestent sous la forme, par exemple, d'une meilleure anticipation des besoins, d'un transfert de connaissances par appropriation préalable chez le constructeur luimême des compétences nécessaires, de montage d'actions de formation en coanimation pendant la phase d'installation entre les moniteurs envoyés par le constructeur et des enseignants d'établissements spécialisés de formation...

Dans tous les cas, les innovations précédemment signalées sont tout à la fois l'expression et le levier d'évolutions plus globales concernant les modes de gestion et de valorisation de la force de travail au sein des PME-PMI (Hillau, 1987). Elles traduisent le besoin de ces entreprises de pouvoir mobiliser des forces de travail plus qualifiées pour faire face aux nouveaux contenus de travail induits par les changements technologiques en cours et pour pouvoir répondre aux nouveaux critères de compétitivité auxquels sont soumises les PME-PMI (tendance à la complexité croissante du travail, besoin de recomposer de nouveaux collectifs de travail, montée des nouvelles professionnalités en amont et en aval de la fabrication, rôle central des savoir-faire d'organisation, de gestion de réseaux...). En ce sens, il y a uniformisation des contraintes en matière de contenu du travail entre grande entreprise et PMEPMI, et nécessité de modeler de nouveaux processus d'apprentissage pour y faire face.

Par ailleurs, l'émergence de pratiques de formation innovantes s'inscrit dans un processus d'insertion plus large des PME-PMI dans des réseaux externes de compétences en matière d'aide, et d'assistance, particulièrement dans le domaine de la formation. Ceci est à rapprocher d'observations faites par ailleurs sur la montée et le développement de nouveaux profils d'entrepreneurs, le recrutement ou l'avènement d'ingénieurs et de gestionnaires qui restent en contact avec leurs anciens réseaux et qui sont capables, compte tenu de leur expérience professionnelle antérieure, de mieux mobiliser les ressources extérieures pour la gestion de leurs ressources humaines.

Enfin les expériences originales en matière de formation s'inscrivent dans les évolutions en cours concernant les modes d'articulation des PME-PMI avec les grands groupes industriels (Raveyre, 1988) et qui se traduisent à la fois par de nouvelles contraintes à l'origine de nouveaux besoins de formation (en particulier pour la mise en place par exemple des procédures de juste à temps ou pour 
l'application des contrats de qualité...) et par de nouvelles relations en terme de formation et d'assistance. Peut-on parler de nouvelles pratiques de partenariat autour d'une composante formation ? Pour l'instant, il est difficile de répondre de manière pertinente à cette question. Notre impression est que ces préoccupations restent le plus souvent au stade du discours, mais que quelques premières expériences, limitées et souvent «clandestines» de la part d'établissements rattachés à des grandes entreprises donneurs d'ordre, commencent à apparaître.

\subsection{Les innovations en matière de gestion externe de la formation des PME-PMI}

Tendanciellement, on observe une cohérence croissante entre les modes de gestion internes et externes de la formation dans le milieu des PME-PMI. Les innovations qui apparaissent dans la mise en oeuvre des politiques de formation au sein de ces entreprises sont souvent permises, facilitées et amplifiées par les changements en cours dans leur environnement, qu'il s'agisse des organismes de formation proprement dit ou des organismes d'aide, d'assistance ou de conseil en matière de formation.

Concernant ces derniers organismes, l'impression qui domine est que l'on a affaire à un marché en pleine mutation autour de trois axes principaux.

En premier lieu on constate un renforcement des organismes «traditionnels» au sens où leur implantation sur le marché de la formation est ancienne (FAF, ASFO en particulier). Dans la période antérieure, ils ont acquis un ensemble de savoir-faire dans le domaine de la formation qui leur permettent d'intervenir de manière beaucoup plus efficace à l'interface entre l'entreprise et l'offre de formation sous la forme d'une diversification de leur domaine de compétences (particulièrement en amont en matière d'analyse de besoin) et d'une intégration croissante entre les différentes modalités d'intervention qu'ils proposent.

En second lieu, l'environnement des politiques de formation des PME-PMI s'est fortement modifié sous la pression des politiques publiques d'emploi et de formation qui ont eu tendance, ces dernières années, à se multiplier, à se spécialiser et à se diversifier. Dans ce contexte, les PME-PMI bénéficient de manière croissante du dispositif des aides publiques en matière d'emploi, en particulier dans sa composante formation, soit directement (ex.: les conventions de développement de la formation ou les procédures FRAC pour la formation) soit indirectement (ex.: aide à la réinsertion, dispositif des nouvelles qualifications, développement de l'alternance...).

Troisièmement, on assiste dans le domaine de l'assistance et du conseil en matière de formation en direction des PME-PMI, à l'émergence de nouveaux acteurs. D'une part, il y a une véritable «explosion» sur ce marché, sous la forme d'un développement du nombre de sociétés ou de consultants qui se lancent dans ces activités. D'autre part, même si le processus reste plus embryonnaire, on signale des 
cas d'interventions originales des collectivités locales en appui au développement des PME-PMI en terme de formation (cas G).

Qu'en est-il des évolutions observables parmi les organismes de formation dans leurs rapports avec les PME? Au départ de cette recherche, nous avons procédé à une large enquête auprès des organismes régionaux qui interviennent en matière de formation dans les PME-PMI. De manière quasi-unanime pour ces différents organismes, le marché des PME-PMI représente un marché colossal d'avenir. En même temps ce marché leur apparaît comme particulièrement difficile d'accès. De fait, ces organismes ne développent pas une offre particulière, spécifique aux PMEPMI. La tendance est plutôt à la recherche d'adaptations, d'infléchissements des formations proposées à la clientèle des grandes entreprises,... ou à l'intégration dans les stages inter-entreprises qu'elles organisent, de stagiaires en provenances des PME-PMI. Pourtant, deux évolutions, plus novatrices, témoignent d'une intégration croissante de la demande de formation des PME-PMI dans l'offre de formation de ces organismes.

D'une part, il y a manifestement une diversification des modes d'intervention de ces organismes dans le sens d'un développement de stages spécifiques, sur mesure, au détriment des formations sur catalogue. D'autre part, les activités de formation s'insèrent dans des modes d'articulation plus diversifiés, plus évolutifs, plus complexes entre les organismes de formation et les entreprises. Il est encore trop tôt pour parler d'un partenariat entre ces acteurs, sauf à propos de quelques cas particuliers. Cependant, un certain nombre de tentatives - même embryonnaires sont significatives de changements en cours à l'interface entre les organismes de formation et les entreprises à travers, par exemple, l'envoi de stagiaires en entreprises, l'organisation de l'alternance, des dispositifs PAE ou des Ateliers professionnels personnalisés.

Enfin, outre la muliplication et la diversification des acteurs qui interviennent dans le domaine de la formation en direction des PME-PMI, on observe des modalités croissantes d'interpénétration et d'imbrication entre ces acteurs.

A ce niveau aussi, il est encore trop tôt pour qualifier ces rapports en terme de partenariat. On a plutôt affaire à la montée de nouvelles solidarités qui se manifestent de manière multiforme. Il en est ainsi par exemple des relations entre les organismes publics et privés de formation, entre les organismes de formation et les organismes de gestion et de conseil, entre ces organismes et les PME-PMI. L'émergence et le développement de ces nouvelles configurations, se traduit par d'importantes conséquences pour l'avenir des enjeux de la formation au sein de ces entreprises.

En effet on assiste à la mise en place de nouveaux systèmes d'apprentissage et de production des qualifications, plus complexes que les précédents dans la mesure où ils mettent en oeuvre un plus grand nombre d'acteurs et selon des modalités plus diversifiées. Ces recompositions multiformes et variées autorisent des modes de 
couverture des besoins plus facilement modulables aux particularités des PME-PMI. Ils vont aussi dans le sens d'une plus grande adaptabilité de l'offre de formation aux spécificités des PME-PMI dans la mesure où les nouveaux espaces de formation apparaissent plus intégrés que les précédents (entre l'organisation de stages et la recherche de financement, entre la formation et l'analyse des besoins, entre la formation et la production dans l'entreprise...).

Au terme de la première phase de la recherche, nos observations de terrain, même limitées, valident notre hypothèse de départ sur l'émergence de pratiques de formation innovantes dans le secteur des PME-PMI. Elles suggèrent en tous cas la convergence de deux familles d'évolution: au sein des PME-PMI et dans leur environnement externe.

D'une part, dans l'environnement des PME, il se développe un réseau de compétences qui associe de nouveaux acteurs et qui tend à couvrir de manière plus formalisée l'ensemble du processus de formation par intégration croissante des activités d'amont (activités d'aide et de conseil pour la définition des besoins, le recours aux financements publics, le choix des organismes de formation). En même temps, l'insertion des PME-PMI dans le fonctionnement de ces réseaux autorise une meilleure adéquation des services rendus (en matière de formation en particulier) aux spécificités des PME-PMI. D'autre part, au sein des PME-PMI, il apparaît des capacités croissantes à mobiliser ces compétences externes en matière d'assistance et de formation, qui compensent le manque ou la faiblesse de compétences internes dans les fonctions de gestion des ressources humaines.

\section{$5 \quad$ Les limites de ces pratiques novatrices}

De toute évidence, la taille de notre échantillon interdit toute généralisation. Elle oblige à un maximum de prudence quant à l'interprétation de ces premières observations. Cela est d'autant plus nécessaire que les innovations que nous avons analysées sont le plus souvent d'origine récente. Leur caractère souvent imparfaitement stabilisé, rend mal aisé une évaluation de leur résultats. rogations.

Toutefois, ces précautions prises, on peut lister une première série d'inter-

La première série d'interrogations concerne l'importance quantitative des PME-PMI innovantes en matière de formation. Même dans le cadre d'une vision extensive de la notion d'innovation, il n'apparaît pas que ces expériences en cours soient quantitativement significatives. D'une manière générale, dans la mesure où l'émergence des nouvelles pratiques de formation est indissociable des changements en cours dans les modalités d'organisation des PME-PMI, ce qui est en cause c'est le caractère non généralisé d'un nouveau modèle de PME-PMI.

En second lieu, notre enquête vérifie que l'une des limites majeures réside dans les difficultés des PME-PMI à définir des stratégies de modernisation et à les 
traduire en terme de besoins de formation pour gérer les processus d'apprentissage correspondants. Majoritairement, la dimension formation reste une variable déterminée, intervenant sur l'impact de décisions prises par ailleurs. Elle n'est qu'exceptionnellement une variable déterminante des changements. Il en résulte différentes limites par rapport à l'efficacité des stages de formation (stages sous la forme de simples adaptations, formations limitées aux seuls opérateurs concernés par la formation...).

En troisième lieu, et dans le prolongement des commentaires précédents, ce qui est en cause c'est la sous-estimation d'un certain nombre de besoins autres que ceux liés à des objectifs directs de production. Il en est ainsi des besoins de formation générale de base pour des publics qui sont souvent détenteurs d'importants savoirfaire mais dont les évolutions sont bloquées par des niveaux de culture générale de base trop faibles. On citera aussi les besoins de formation dans les domaines de la gestion de production et de l'industrialisation, où les compétences disponibles au plan interne sont souvent les plus faibles alors qu'elles constituent un enjeu qui aura une importance croissante dans les prochaines années.

Une quatrième série de limites concerne les modalités d'élaboration et de conception des politiques de formation, et en particulier, les conditions de participation de salariés. Ces derniers apparaissent généralement exclus de ces procédures, ce qui conduit les PME-PMI à se priver d'une capacité d'expertise tout à fait essentielle à la définition de leurs besoins de formation.

Une cinquième série de limites concerne l'environnement des PME-PMI en matière de formation. Outre les problèmes bien connus en terme de coordination, de transparence et d'information, notre enquête suggère deux séries de commentaires. La première concerne le système d'aide mis en place par les pouvoirs publics. Par delà la diversité et l'hétérogénéité du dispositif, celui-ci semble plus orienté vers un appui aux PME-PMI dynamiques qu'aux autres. Dans ces conditions, le risque est grand qu'il conduise à un renforcement des disparités au sein du secteur, plutôt qu'à égaliser les chances d'accès à la formation. Autrement dit, ce qui est en cause, c'est la situation des PME-PMI quin'ont pas encore démarré un processus d'apprentissage à la gestion des problèmes de formation et qui, de fait, sont exclues du dispositif d'aide.

La seconde famille de commentaires conceme plutôt les expériences novatrices décrites précédemment. Les actions qu'elles développent témoignent de l'intérêt des préoccupations qui les sous-tendent. Elles reposent sur des intuitions et des objectifs dont on a signalé le caractère positif. La question qui se pose est celle des conditions de leur mise en oeuvre et surtout de leur généralisation et de la correspondance entre ces principes et leurs réalisations. 


\section{BIBLIOGRAPHIE}

Mehaut, (1989), «Vers un nouveau rapport de l'entreprise à la formation ?», G.R.E.E./CNRS, Principales conclusions de la recherche «Production et usage de la formation par et dans l'entreprise», p. 28.

Gehin, (1986), «La formation continue dans les PME: spécificités et paradoxes», Formationemploi, $\mathrm{n}^{\circ}$ 16, CEREQ, pp. 77-91.

Lutz, (1983), «Problèmes de la formation professionnelle dans les PME», Actualités de la Formation permanente, $\mathrm{n}^{\circ} 67$, pp. 41-47.

Fougère, (1986), «Gestion de la main-d'oeuvre et formation dans les PMI automatisées», Formation-emploi, $\mathrm{n}^{\circ}$ 16, CEREQ, pp. 70-76.

Cf. aussi le compte rendu des travaux de la conférence française EUROTECNET, 19 et 20 mai 1988. L'investissement formation dans les PME, 1989 ADEP, p. 168.

Hillau, (1987), «PME, gestion du personnel et environnement de l'entreprise», Formationemploi, ${ }^{\circ} 19$, CEREQ, p. 19-24.

Raveyre, (1988), «Jeux de miroirs. L'aide de St-Gobain développement aux PME», GLYSIPIRTTEM, p. 240. 